Document downloaded from:

http://hdl.handle.net/10251/144585

This paper must be cited as:

Godoy-Reyes, TM.; Costero, AM.; Gaviña, P.; Martínez-Máñez, R.; Sancenón Galarza, F. (22-0). A Colorimetric Probe for the Selective Detection of Norepinephrine Based on a Double Molecular Recognition with Functionalized Gold Nanoparticles. ACS Applied Nano Materials. 2(3):1367-1373. https://doi.org/10.1021/acsanm.8b02254

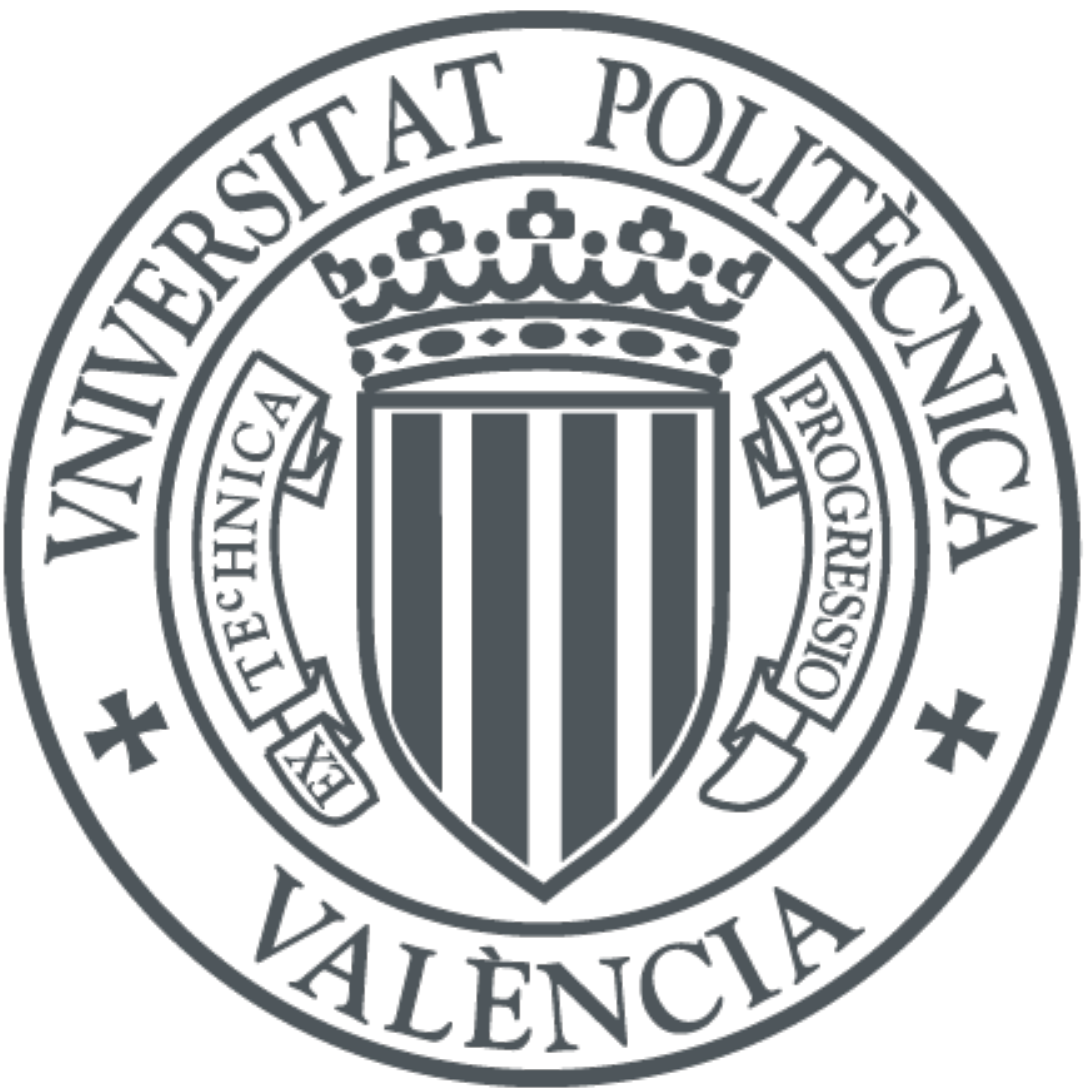

The final publication is available at

https://doi.org/10.1021/acsanm.8b02254

Copyright American Chemical Society

Additional Information 


\title{
A Colorimetric Probe for the Selective Detection of Norepinephrine based on a Double Molecular Recognition with Functionalized Gold Nanoparticles
}

\author{
Tania M. Godoy-Reyes, ${ }^{\dagger, \neq, \|}$ Ana M. Costero, ${ }^{\dagger, \ddagger, \S}$ Pablo Gaviña, ${ }^{*, t, \neq, \S}$ Ramón Martínez- \\ Máñez ${ }^{*,+, \neq, \|}$ and Félix Sancenón ${ }^{\dagger, \ddagger, \|}$

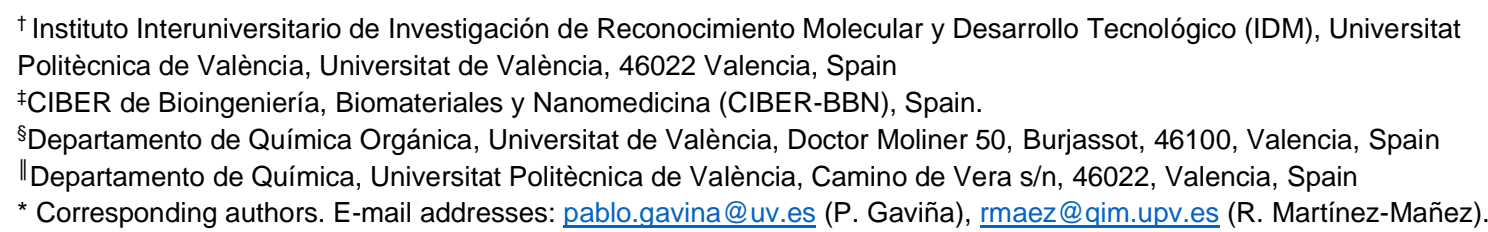

Abstract: A simple colorimetric probe for the selective and sensitive detection of neurotransmitter norepinephrine (NE), an important biomarker in the detection of tumors such as pheochromocytoma and paraganglioma, is described. The sensing strategy is based on the use of spherical gold nanoparticles functionalized with benzaldehyde and boronic acid-terminated moieties. A double molecular recognition involving on the one hand the aromatic aldehyde and the aminoalcohol group of NE, and on the other hand the boronic acid and the catechol moiety of the neurotransmitter, results in analyte triggered aggregation of the gold nanoparticles, leading to a bathochromic shift of the SPR band in the UV-vis spectrum of the probe and a clear change in the color of the solution from red to blue. Probe P1 shows a remarkable selectivity towards NE versus other catecholamine neurotransmitters (dopamine and epinephrine) and selected biomolecules (5-HIAA, L-Tyr, glucose, uric acid, Lys and glutamic acid). Moreover, a linear response to $\mathrm{NE}$ in the $0-1 \mu \mathrm{M}$ concentration range was observed and limit of detection of $0.07 \mu \mathrm{M}$ in aqueous media was determined by UV-vis spectroscopy. The sensitivity of the probe towards $\mathrm{NE}$ in synthetic urine was also evaluated. In this medium, a limit of detection of $0.09 \mu \mathrm{M}$ was obtained which falls within the range of clinical interest.

Keywords: Norepinephrine (NE) • neurotransmitters • gold nanoparticles • colorimetric detection • aggregation 


\section{Introduction}

Norepinephrine (NE) or noradrenaline, also known as the "hormone of the stress" is a hormone and a neurotransmitter secreted by noradrenergic neurons in the sympathetic and central nervous system. NE plays important roles in physiological processes such as stress, [12] anxiety, [3-4] sleep, [5] and memory. Additionally, NE is involved in the fight-or-flight response, triggering the release of glucose and increasing heart rate and blood flow to skeletal muscle. [7] Furthermore, it also regulates the function of certain types of immune cells (e.g., T and NK cells).[8] Pathologies such as depression,[9-10] Alzheimer's disease,[11] and Parkinson's disease are associated with low levels of NE.[12] Conversely, high NE levels are indicative of pheochromocytoma and paraganglioma, two neuroendocrine tumors, which start in the adrenal medulla and extra-adrenal paraganglia respectively and secrete large amounts of NE.[13-14] This increases NE levels in patients' urine and causes hypertension, fast heart rate, headache, sweating and chest or abdominal pain.[15] In addition, human immunodeficiency virus-1 (HIV-1) replication is accelerated by NE via protein kinase A-dependent effects on cytokine production.[16]

Given the importance of NE as biomarker for the early diagnosis of the previously commented pathologies, the development of effective and simple methods for the detection and quantitative determination of NE is highly desirable.

In recent years, some analytical methods have been developed for the determination of NE, such as high performance liquid chromatography (HPLC), [17-18] fluorometric [19-20] and electrochemical techniques [21-23]. Although these reported methods are able to determine correctly the presence of $\mathrm{NE}$, in general expensive equipment and complicated sample pretreatment is needed. Hence, the development of fast, low cost, sensitive and selective techniques for the specific and simple detection of NE in biological fluids is of great importance.

The use of chromogenic sensors and probes is an appealing alternative to overcome some limitations of traditional instrumental methods, because their low cost and operational simplicity, allow on-site detection, and require simple equipment or no instrumentation at all [24]. Remarkably in spite of them being of much interest, very few colorimetric methods have been developed to detect NE [25-30]. Moreover, most of these methods are based on the use of 
catecholamines as reducing agents, which lead to reduced selectivity to NE in the presence of other catecholamines, such as dopamine or epinephrine (see Table S3 Supporting Information).

Gold nanoparticles (AuNPs) are excellent materials, which have been successfully applied for the design of colorimetric probes. [31-33] In general, the detection strategy relies on the observed color changes that arise upon analyte-triggered aggregation or the redispersion of nanoparticles. Colloidal solutions of dispersed AuNPs, a with particle size between 5-30 nm, exhibit a deep red color and a high molar extinction coefficient due to a surface plasmon resonance (SPR) band. Moreover, AuNPs can be easily functionalized on their surface with a broad range of organic molecules or biological macromolecules, which selectively interact with the target analyte. This interaction triggers the aggregation of AuNPs, resulting in interparticle surface plasmon coupling. This results in an important bathochromic shift of the SPR band and a change in the color of the solution from red to blue, which can be seen with the naked eye at very low analyte concentrations. [34-37] For instance, the implementation of double-functionalized AuNPs as probes for the selective colorimetric detection of neurotransmitters such as dopamine [38] or serotonin [39] has been previously reported.

Based on the aforementioned facts, and following our interest in developing colorimetric methods for the fast, simple and selective detection of biomarkers, we report herein the use of bifunctionalized AuNPs as probes for the colorimetric detection of NE, through the double molecular recognition of the catechol and aminoalcohol moieties.

The sensing strategy is sketched in Scheme 1. Probe P1 consists of AuNPs (ca. $14 \mathrm{~nm}$ ), functionalized on their surface with two different ligands, 4-(liponyloxy)benzaldehyde (L1) and 4mercatophenylboronic acid (L2). The terminal aldehyde of L1 was chosen to react with the aminoalcohol group of NE to form an oxazolidine,[40,41] whereas L2 plays a double role: (i) the boronic acid group is able to react with the catechol moiety in NE to form stable boronate groups [42-43]; (ii) the ligand also acts as a stabilizer for AuNPs. A double interaction between NE and the dispersed gold nanoparticles was expected to trigger their aggregation leading to a shift of the AuNPs SPR band, with the resulting change in the color of the solution (from red to blue).

Scheme 1. Sensing strategy for the colorimetric detection of norepinephrine (NE) using gold nanoparticles functionalized with L1 and L2 (P1). 


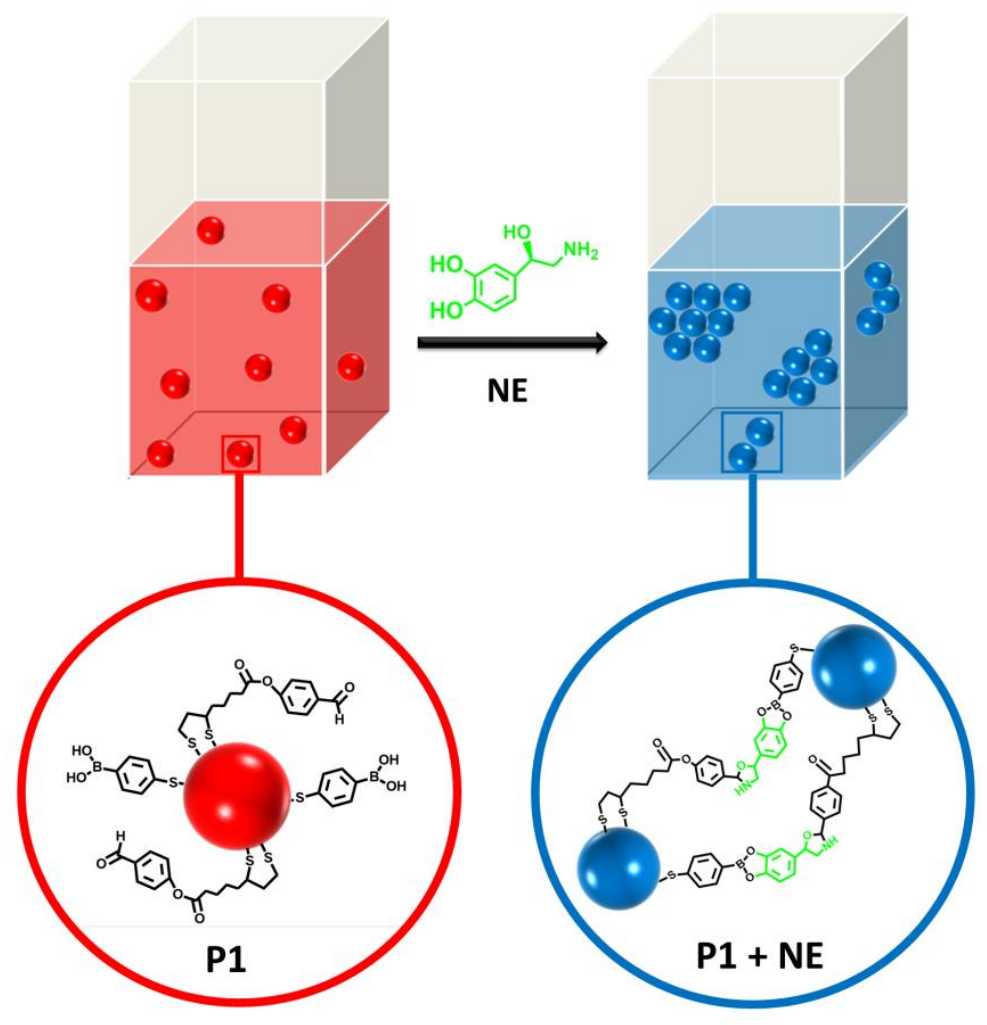

\section{Experimental}

\subsection{Synthesis of probe $\boldsymbol{P 1}$}

Probe P1 was synthesized from freshly prepared citrate-stabilized gold nanoparticles of ca. 14 $\mathrm{nm}$ diameter (see Supp Info) by a ligand-exchange reaction with L1 and L2, which was performed as follows: First $\mathrm{L} 2(20 \mu \mathrm{l}, 0.5 \mathrm{mM}$ in DMF) was added to an aqueous dispersion of the asprepared citrate-coated AuNPs $(10 \mathrm{~mL})$ and the mixture stirred for $1 \mathrm{~min}$ at room temperature. Then L1 (20 $\mu \mathrm{l}, 0.5 \mathrm{mM}$ in DMF) was added to the solution and the mixture was stirred at room temperature for $1 \mathrm{~h}$. The resulting suspension of nanoparticles was centrifuged (10 min, 11000 rpm), the supernatant was decanted and the nanoparticles redispersed again in Mili-Q water (18.2 $\left.\mathrm{M} \Omega \mathrm{cm}^{-1}\right)$. This procedure was repeated twice to yield probe $\mathbf{P 1}$.

\subsection{Sensing studies}

NE solutions of different concentrations were freshly prepared at $\mathrm{pH} 6.5$ in $10 \mathrm{mM}$ phosphate buffer at room temperature. Then $200 \mu \mathrm{L}$ of the NE solution at the corresponding concentration was added to $200 \mu \mathrm{L}$ of the solution of $\mathbf{P} 1\left(8.1 \times 10^{-10} \mathrm{M}\right)$. The resulting mixture of NE and probe 
P1 $(400 \mu \mathrm{L})$ was incubated for $5 \mathrm{~min}$ and then the corresponding UV-vis spectra was recorded at room temperature. The same protocol was followed in the study of interferences.

The assays run with the optimized probe (OP1) were performed by adding $20 \mu \mathrm{L}$ of NE (500 $\mu \mathrm{M}$, final concentration) to $200 \mu \mathrm{L}$ of $\mathbf{P 1}$ and incubating for 1 minute. Next $180 \mu \mathrm{L}$ of the NE problem solution at different concentrations were added resulting in a final volume of $400 \mu \mathrm{L}$. UV-vis measurements were performed after an incubation time of $5 \mathrm{~min}$. In order to ensure that the priming of the probe does not bias the results, $220 \mu \mathrm{L}$ of the mother solution of OP1 plus $180 \mu \mathrm{L}$ of phosphate buffer is used as blank ( 0 added NE) and compared with the signal of the optimized probe upon addition of external NE. This procedure of priming gold nanoparticles-based probes has been carried out based on previously reported studies. [38]

Surine $^{\mathrm{TM}}$ (synthetic urine negative control) was used for the measurements of NE in urine. To take these measurements, OP1 $(200 \mu \mathrm{L})$ and $200 \mu \mathrm{L}$ of the NE solutions in $10 \%$ of the synthetic urine were mixed, incubated for $5 \mathrm{~min}$, and their UV-vis spectra were recorded.

\section{Results and Discussion}

Probe P1, which is depicted in Scheme 1, consists of spherical gold nanoparticles functionalized with 4-(liponyloxy)benzaldehyde (L1) and 4-mercaptophenyl boronic acid (L2), which are attached to the AuNPs' surface through the formation of stable Au-S bonds.

L1 was prepared from lipoic acid and p-hydroxybenzaldehyde in the presence of DCC and DMAP with an overall $86 \%$ yield (Scheme S1).

The bifunctionalized AuNPs were prepared as follows: First citrate-capped AuNPs were synthesized by reduction of chloroauric acid with sodium citrate in boiling water, following the Turkevich-Frens method. [44-46] Then, citrate was displaced from the surface of the nanoparticles, in a ligand exchange reaction, by the addition of L1 and L2 (1:1 molar ratio). A repeated process of centrifugation, elimination of the supernatants and redispersion in water yielded probe $\mathbf{P 1}$.

In order to characterize $\mathbf{P 1}$, transmission electron microscopy (TEM), dynamic light scattering (DLS), Fourier-transform infrared spectroscopy (FTIR), inductively coupled plasma mass spectrometry (ICP-MS) and UV-Vis spectroscopy measurements were performed. 
Monodisperse AuNPs with an average size of $14 \pm 2 \mathrm{~nm}$ were observed by TEM (Figure S2). The hydrodynamic diameter of nanoparticles, determined by DLS, increased from $16.7 \mathrm{~nm}$ for the citrate-stabilized AuNPs to $20.5 \mathrm{~nm}$ for the bifunctionalized AuNPs P1 (Figure S3 A). The double molecular functionalization was confirmed by FTIR spectra, which showed the presence of absorption bands corresponding to L1 and L2 (see Figure S5). Finally, the amount of ligands L1 and L2 per gold nanoparticle was calculated from ICP-MS studies (B and S) resulting in ca. 1400 and 930 molecules respectively, corresponding to a L1/L2 molar ratio of 1.5:1 (Table S1).

The aqueous suspensions of $\mathbf{P} \mathbf{1}$ presented the characteristic red wine color and their UVvis spectrum showed the presence of an intense SPR peak at $523 \mathrm{~nm}$ in the, typical of AuNPs with an average size smaller than $30 \mathrm{~nm}$ [47-48]. A final concentration of the P1 nanoparticles of ca. $8.12 \times 10^{-10} \mathrm{M}$ was calculated from their UV-vis spectra, considering a molar extinction coefficient $\varepsilon=3.15 \times 10^{8} \mathrm{M}^{-1} \mathrm{~cm}^{-1}[49]$.

The suspensions of $\mathbf{P 1}$ were stored in the refrigerator until their use. No changes in the characteristic SPR absorption band or in the color of the solutions were observed after more than 3 weeks, which evidences the good stability of P1.

Before starting the sensing studies with $\mathbf{P 1}$, a preliminary ${ }^{1} \mathrm{HNMR}$ study of a mixture of $\mathrm{NE}$ and 4-methoxybenzaldehyde in $\mathrm{MeOH} / \mathrm{H}_{2} \mathrm{O}$ at room temperature confirmed the reaction of the aromatic aldehyde with the aminoalcohol group of NE (see Fig S17 in Supplnfo).

The sensing capability of probe P1 to NE was evaluated in buffered aqueous solution at pH 6.5 (phosphate, $10 \mathrm{mM}$ ) because a preliminary screening showed that this $\mathrm{pH}$ gave the best results. Thus when NE (1000 $\mu \mathrm{M})$ was added to a red-colored suspension of $\mathbf{P} \mathbf{1}$, a clear color change, from red to blue was rapidly observed, and indicated the aggregation of the gold nanoparticles. A remarkable shift of the SPR band from 523 to $621 \mathrm{~nm}$ was observed in the UVvis absorption spectrum (Figure 1) due to aggregation-induced interparticle plasmon coupling. Aggregation of nanoparticles was also confirmed by TEM (Figure S2 A and B) and DLS. The DLS studies showed an increase in the hydrodynamic diameter of the nanoparticles from $20.51 \mathrm{~nm}$ for P1 to $658.8 \mathrm{~nm}$ after the addition of NE (Figure S3 B). Moreover, the Zeta potential lowered from $-28 \mathrm{mV}(\mathbf{P} \mathbf{1})$ to $-12 \mathrm{mV}(\mathbf{P} 1+\mathrm{NE})$ due to the reaction of the recognition moieties with the neurotransmitter and to the aggregation of the nanoparticles (Figure S4). The kinetic studies 
showed a marked increase in the absorbance of $\mathbf{P 1}$ at $621 \mathrm{~nm}$ after the addition of NE since minute 1 , reaching its maximum point in around 5 min (Figure S6).

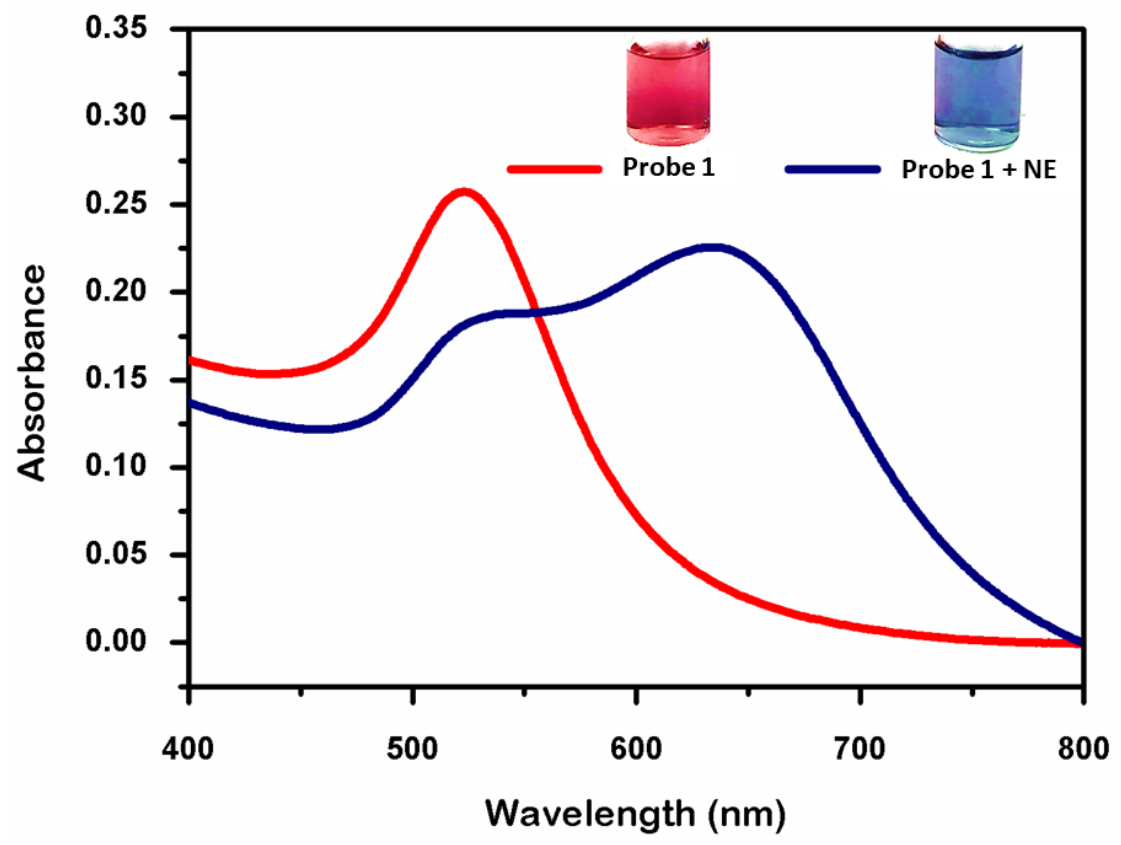

Figure 1. The UV-Vis spectra of $\mathbf{P} 1$ in the absence (red) and in the presence (blue) of $N E(1000 \mu \mathrm{M})(\mathrm{pH} 6.5$ phosphate buffer, $10 \mathrm{mM})$.

The selectivity of $\mathbf{P 1}$ toward NE versus other similar neurotransmitters, such as dopamine (DA), and epinephrine (Epi), and other biomolecules, e.g., 5-hydroxyindoleacetic acid (5-HIAA, the main metabolite of serotonin in urine), L-tyrosine (L-Tyr, precursor of some stimulatory neurotransmitters), glucose (Glc), uric acid (UA, a potential neurotransmitter), lysine (Lys) and glutamic acid (GA, excitatory amino acid) was also evaluated. Upon the addition of these potential interferences to the suspension of $\mathbf{P} \mathbf{1}$, negligible changes in the color or in the $A_{621} / A_{523}$ ratio $\left(A_{621}\right.$ $=$ absorbance at $621 \mathrm{~nm}, A_{523}=$ absorbance at $523 \mathrm{~nm}$ ) of the solutions were observed (Figures 2 and S7, S8). Finally, a competitive experiment was performed to evaluate the response of P1 in a mixture containing NE and DA, Epi, L-Tyr, Gcl, UA, Lys, and GA (1000 $\mu \mathrm{M}$ of each). As shown in Figure 2b, a similar response to that found for $\mathbf{P 1}$ in the presence of NE alone was observed. The stability of $\mathbf{P 1}$ and its selectivity to NE remained almost unchanged within the $6.5-7.5 \mathrm{pH}$ range (Figure S9). We attribute this higher selectivity of the probe towards NE versus other similar catecholamines such as $\mathrm{Da}$ and Epi, to the presence of the aminoalcohol group in NE, which 
allows the formation of a highly stable oxazolidine upon reaction with the aromatic aldehyde attached to the gold nanoparticles. Imine formation is highly unfavourable in water and this would explain the fact that we did not observe aggregation in the presence of dopamine which lacks of the hydroxyl group. On the other hand, the lower response of the probe towards epinephrine under the tested reaction conditions could be a kinetic effect due to the presence of the methyl group attached to the nitrogen atom. In fact, as observed in Figure 2, epinephrine also produces some aggregation of the nanoparticles although to a much lesser extent.

a)

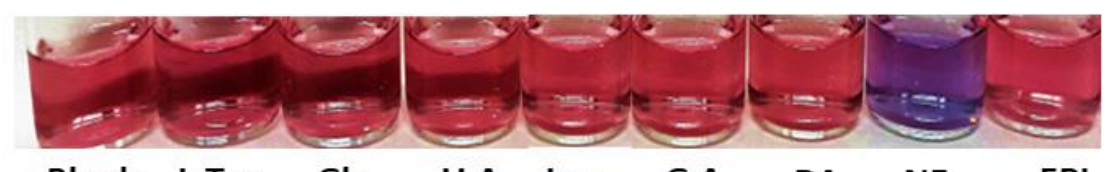

b)
Blank L-Tyr
Glc
U.A Lys
G.A
DA NE
EPI

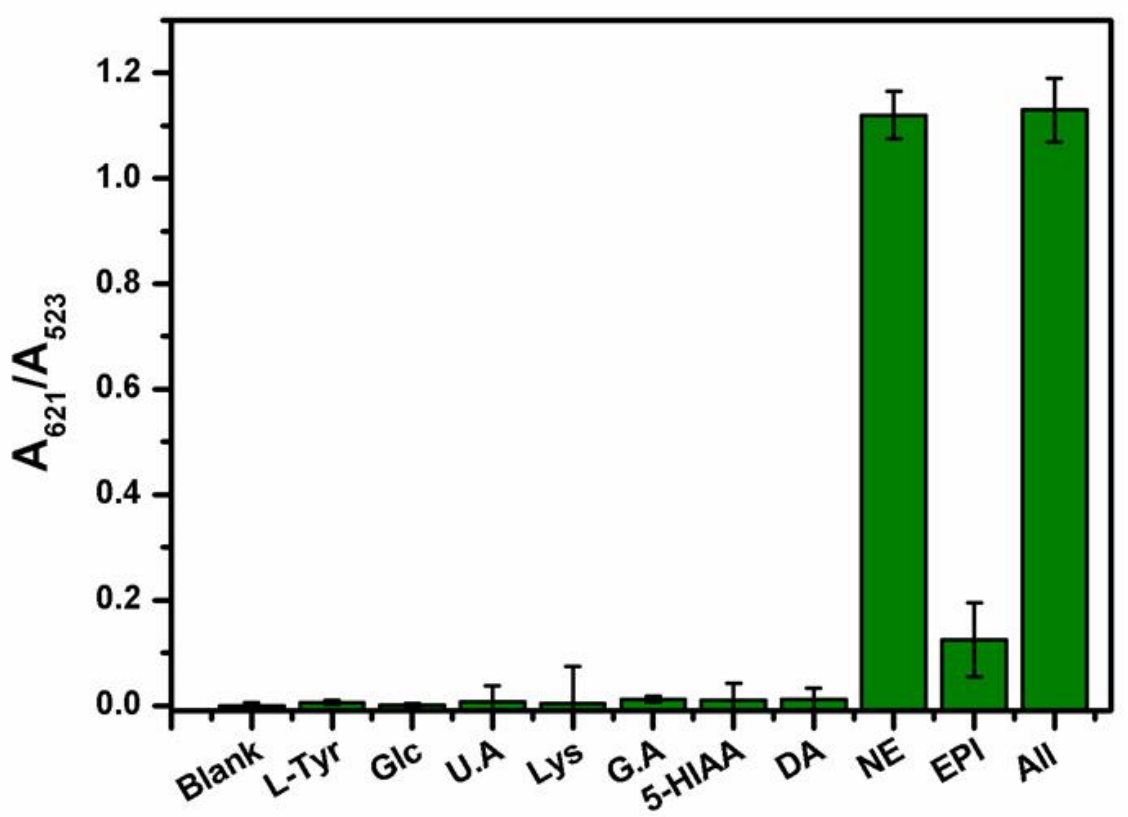

Figure 2. Response of probe P1 toward relevant biomolecules (1000 $\mu \mathrm{M})$ at pH 6.5 (phosphate buffer, $10 \mathrm{mM})$. a) Observed colour changes. b) Representation of $A_{621} / A_{523}$ for NE, the interferents and a mixture of all.

Additionally, control experiments with citrate-capped AuNPs and with AuNPs modified only with L1 (L1-AuNPs) or with L2 (L2-AuNPs) (see Supp. Info for details) were also performed. We observed the aggregation of the citrate-AuNPs in the presence of excess of all the catecholamines $(1000 \mu \mathrm{M})$ [50], whereas negligible changes in the color or in the $A_{621} / A_{523}$ ratio of the solutions were observed upon the addition of an excess of NE, EPI or DA to the suspensions of L1-AuNPs or L2-AuNPs. Finally, the response observed in the $A_{621} / A_{523}$ ratio for an equimolecular mixture 
of L1-AuNPs and L2-AuNPs in the presence of NE $(100 \mu \mathrm{M})$ was much lower than that observed in the presence of P1 (see Supporting Information, Fig S13).

In order to evaluate the sensitivity of probe $\mathbf{P 1}$, UV-vis titration experiments were performed in the presence of increasing amounts of NE. As observed in Figure 3a, the addition of NE to an aqueous suspension of $\mathbf{P} \mathbf{1}$ induced a gradual color modulation that went from clear red to purple, and finally to blue. The corresponding bathochromic shift of the SPR absorption band of P1 with increasing NE concentrations was also observed in the corresponding UV-vis spectra (Figure 3b), and can be attributed to the analyte-induced aggregation of nanoparticles. From the $A_{621} / A_{523} v s$. the $N E$ concentration plot, a limit of detection (LOD) of $1.16 \mu \mathrm{M}$ was determined (Figure 4b curve 2 and Figure S14).

a)

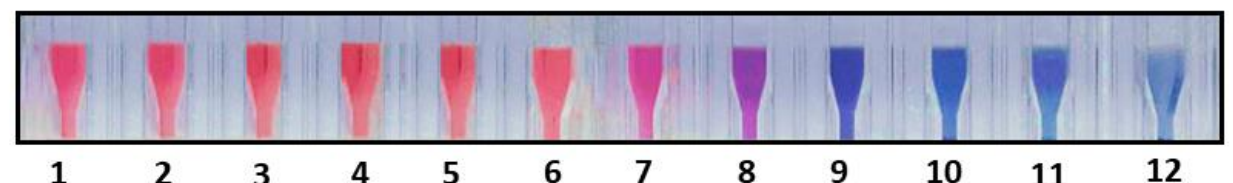

b)

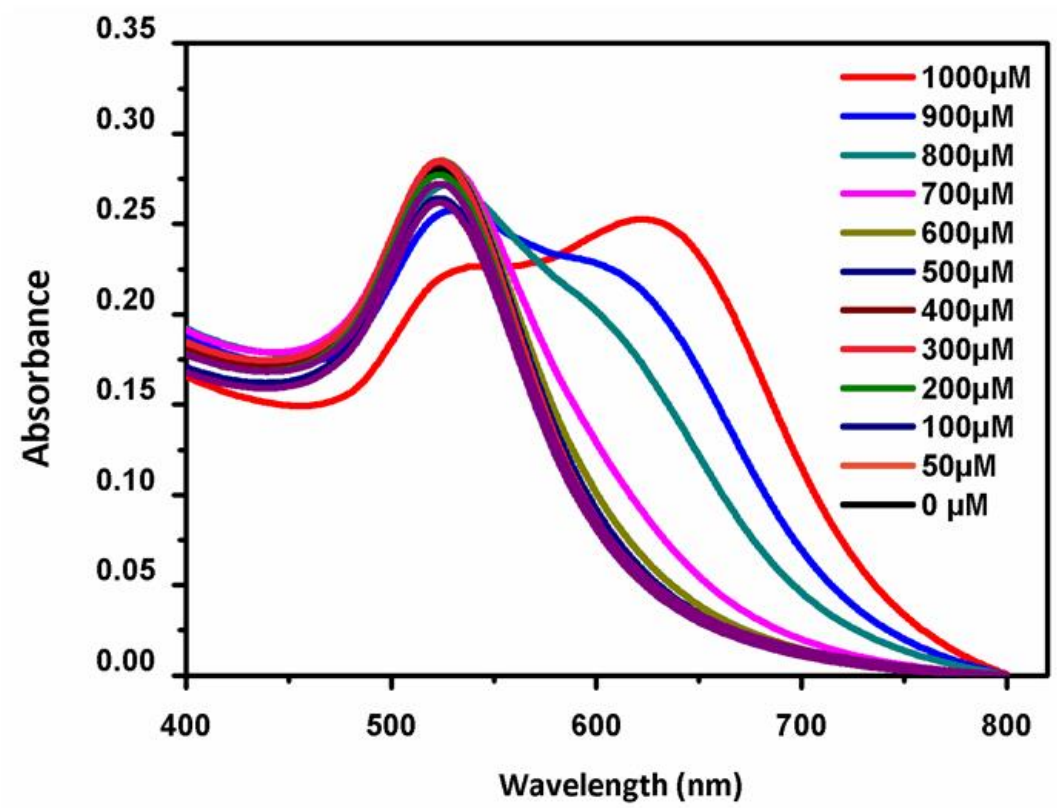

Figure 3. a) Color variation of $\mathbf{P} 1$ after the addition of $\mathrm{NE}$ at the $0,50,100,200,300,400,500,600,700,800,900$ and $1000 \mu \mathrm{M}$ concentrations (1-12, respectively). b) Changes in the UV-Vis spectra of P1 upon addition of NE at $0,50,100$, 200, 300, 400, 500, 600, 700, 800, 900 and $1000 \mu \mathrm{M}$ concentrations at pH 6.5 (phosphate buffer, $10 \mathrm{mM}$ )

As shown in Figure $4 a$, a slight linear increase in the $A_{621} / A_{523}$ ratio with increasing $N E$ concentrations was observed within the 0-500 $\mu \mathrm{M}$ concentration range. Interestingly, a much 
more marked intensity increase was observed within the $500-1200 \mu \mathrm{M}$ concentration range. This fact allowed to improve the sensitivity to NE detection by simply adding a certain fixed amount of NE to the stock solution of $\mathbf{P 1}$ immediately before use. [38] Thus an optimized probe (OP1) was prepared by adding $500 \mu \mathrm{M}$ of $\mathrm{NE}$ to the $\mathbf{P} 1$ solution in advance. As observed in the $A_{621} / A_{523} v s$. the NE concentration plot for OP1 (Figure 4b, curve 1), sensitivity is greater than it is for P1. From this plot, a LOD of $0.07 \mu \mathrm{M}$ could be determined (see Figure S15). This low LOD of OP1 is indicative of the remarkable sensitivity of the optimized probe, conferring the system the possibility of detecting NE at relevant clinical concentrations.
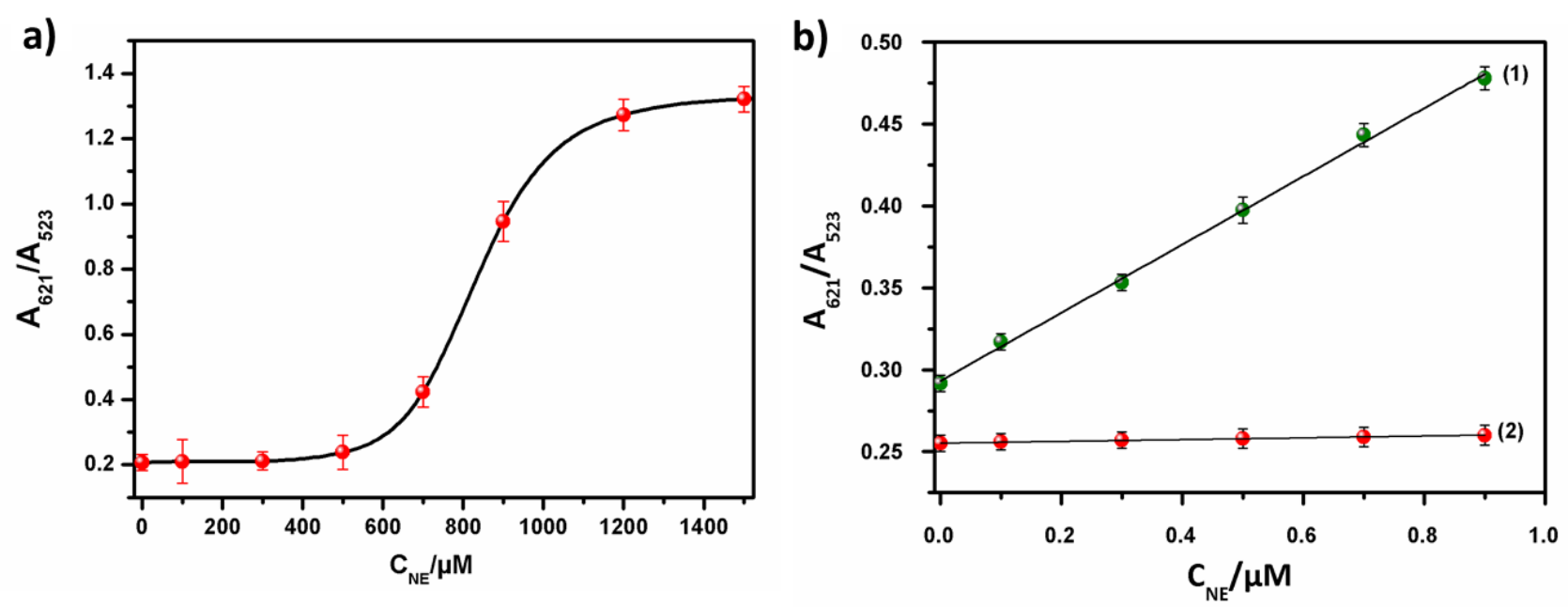

Figure 4. a) Plot of $A 621 / A 523$ versus NE concentration (0-1500 $\mu \mathrm{M})$ for $\mathbf{P 1}$. b) Linear plots of $A 621 / A 523$ versus the NE concentration obtained with (1) OP1 and (2) P1.

The normal range for norepinephrine in healthy individuals is 0.08 to $0.47 \mu \mathrm{M}$ in urine. Reported studies determined that higher NE levels could be indicative of pheochromocytoma and paraganglioma tumors. [51] Given the interest in the determination of NE concentration in urine, we decided to test the response of OP1 in this highly competitive media.[52] OP1 in urine showed a similar behavior to that described before in aqueous media upon the addition of increasing concentrations of NE (Figure $S 16$ ). The plot of $A_{621} / A_{523}$ vs. NE concentration in urine shows a linear response within the $0-1 \mu \mathrm{M}$ concentration range (Figure 5). The determined LOD in this medium was $0.09 \mu \mathrm{M}$, which falls within the normal range for adults of 0.08 to $0.4 \mu \mathrm{M}$ in urine. [51] 


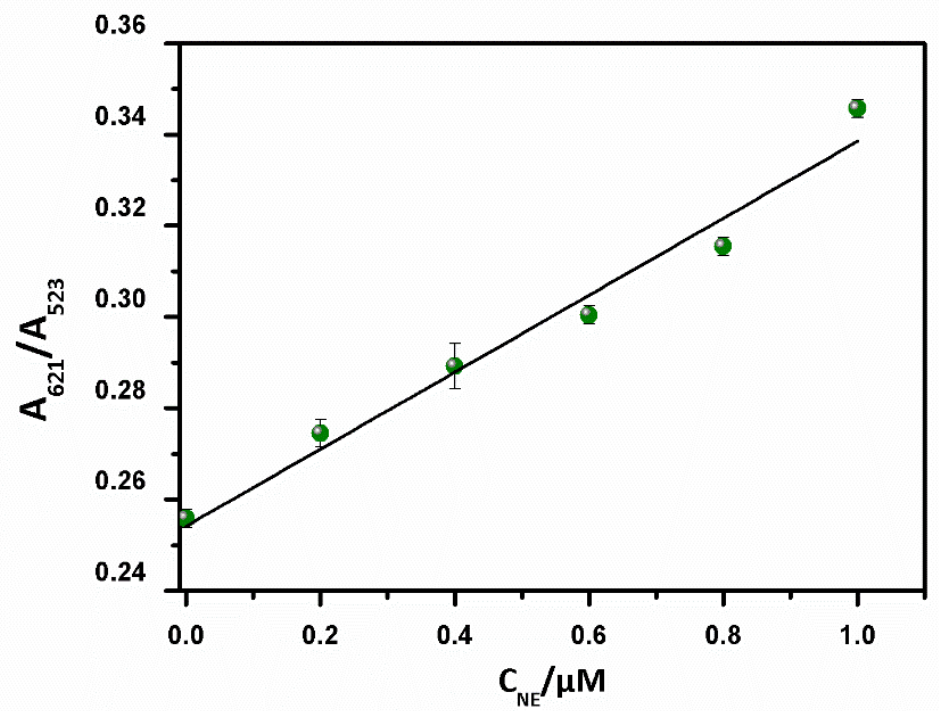

Figure 5. Linear plot of A621/A523 versus the NE concentration obtained with OP1 in urine samples

\section{Conclusions}

In short, we have developed a new approach for the selective and sensitive colorimetric detection of NE using bifunctionalized gold nanoparticles based on a double recognition process. Probe P1, consisting of AuNPs functionalized with boronic acid and aldehyde-terminated ligands, selectively reacted with the catechol group and the aminoalcohol moiety of NE in aqueous buffered solution, and led to nanoparticle aggregation. This resulted in a remarkable color change in the solution from red to blue, and the appearance of a new absorption band at $621 \mathrm{~nm}$ in the UV-vis spectrum. Due to this double interaction, $\mathbf{P 1}$ showed excellent selectivity to NE, whereas almost no response of the probe was observed for similar neurotransmitters (dopamine, and epinephrine) or selected biomolecules (5-hydroxyindoleacetic acid, L-tyrosine, glucose, uric acid, lysine and glutamic acid). An optimized probe (OP1) was prepared by adding a certain fixed amount of NE to the probe before using it. OP1 showed a linear response to NE within the $0-1$ $\mu \mathrm{M}$ range in buffered water at $\mathrm{pH} 6.5$, with a calculated LOD of $0.07 \mu \mathrm{M}$. A similar response to NE in synthetic urine could be observed, with a LOD in this medium of $0.09 \mu \mathrm{M}$. To conclude, our system allows the rapid, cheap, selective and sensitive detection of NE by overcoming some of 
the limitations of current methods. We think that our findings could help to early diagnoses diseases such as pheochromocytoma or paraganglioma for which NE is an important biomarker.

\section{Acknowledgments}

Financial support from the Spanish Government (Projects MAT2015-64139-C4-1-R and MAT2015-64139-C4-4-R) and the Generalitat Valencia (Projects PROMETEOII/2014/047 and AICO/2017/093) is gratefully acknowledged. T. Godoy-Reyes is grateful to the Generalitat Valenciana for her Santiago Grisolía fellowship. SCSIE (Universitat de València) is gratefully acknowledged for all the equipment employed.

\section{Supporting Information}

The supporting information associated with this article is available free of charge at...

Chemicals and general procedures. Synthesis and characterization of L1 and probe P1 (NMR, IR, TEM, DLS), pH and kinetic studies, UV-vis spectra of P1 in the presence of interferents, synthesis characterization and evaluation of the colorimetric response of L1-AuNPs and L2AuNPs, determination of limits of detection, recovery and accuracy of the method, comparison with other methods, response of $\mathrm{P} 1$ in urine and ${ }^{1} \mathrm{H}-\mathrm{NMR}$ experiments.

\section{References}

[1] Nakane, H.; Shimizu N.; Hori, T., Stress-Induced Norepinephrine Release in the Rat Prefrontal Cortex Measured by Microdialysis, Am. J. Physiol. Regul. Integr. Comp. Physiol. 1994, 267, R1559$\mathrm{R} 1566$

[2] Simeon, D.; Knutelska, M.; Smith, L.; Baker, B. R.; Hollander, E., A Preliminary Study of Cortisol and Norepinephrine Reactivity to Psychosocial Stress in Borderline Personality Disorder with high and low Dissociation, Psychiatry Res. 2007, 149, 177-184.

[3] Spivak, B.; Vered, Y.; Graff, E.; Blum, I.; Mester R.; Weizman, A., Low Platelet-poor Plasma Concentrations of Serotonin in Patients with Combat-related Posttraumatic Stress Disorder, Biol. Psychiatry 1999, 45, 840-845. 
[4] Goddard, A. W.; Ball, S. G.; Martinez, J.; Robinson, M. J.; Yang, C. R.; Russell, J. M.; Shekhar, A., Current Perspectives of the Roles of the Central Norepinephrine System in Anxiety and Depression, Depress. Anxiety, 2010, 27, 339-350

[5] Hunsley, M. S.; Palmiter, R. D., Norepinephrine-Deficient Mice Exhibit Normal Sleep-wake States but have Shorter Sleep Latency after Mild stress and low Doses of Amphetamine, Sleep 2003, 26, 521-526.

[6] Murchison, C. F.; Zhang, X. Y.; Zhang, W. P.; Ouyang, M.; Lee, A.; Thomas, S. A., A Distinct Role for Norepinephrine in Memory Retrieval, Cell, 2004, 117, 131-143.

[7] Jansen, A. S.; Van Nguyen, X.; Karpitskiy, V.; Mettenleiter, T. C.; Loewy, A. D., Central Command Neurons of the Sympathetic Nervous System: Basis of the Fight-or-Flight Response, Science, 1995 , 270, 644-646.

[8] Takenaka, M. C.; Araujo, L. P.; Maricato, J. T.; Nascimento, V. M.; Guereschi, M. G.; Rezende, R. M.; Quintana F. J.; Basso, A. S., Norepinephrine Controls Effector T Cell Differentiation through $\beta 2-$ Adrenergic Receptor-Mediated Inhibition of NF-kB and AP-1 in Dendritic Cells, J. Immunol. 2016, 196, 637-644.

[9] Jobe, P.C.; Dailey J.W.; Wernicke, J.F., A Noradrenergic and Serotonergic Hypothesis of the Linkage between Epilepsy and Affective Disorders, Crit. Rev. Neurobiol. 1999, 13, 317-356

[10] Nutt, D. J.; The Role of Dopamine and Norepinephrine in Depression and Antidepressant Treatment, J Clin Psychiatry, 2006, 67, 3-8.

[11] Chalermpalanupap, T.; Kinkead, B.; Hu, W. T.; Kummer, M. P.; Hammerschmidt, T.; Heneka, M. T.; Weinshenker D.; Levey, A. I., Targeting Norepinephrine in Mild Cognitive Impairment and Alzheimer's Disease, Alzheimers Res. Ther. 2013, 5, 21-29.

[12] Delaville C.; De Deurwaerdère, P.; Benazzouz, A., Noradrenaline and Parkinson's Disease, Front. Syst. Neurosci. 2011, 5, 31-42.

[13] Higashi, Y.; Sasaki, S.; Nakagawa, K.; Kimura, M.; Sasaki, S.; Noma, K.; Matsuura, H.; Hara, K.; Goto, C.; Oshima T.; Chayama, K., Excess Norepinephrine Impairs both Endothelium-Dependent and-Independent Vasodilation in Patients with Pheochromocytoma, Hypertension, 2002, 39, $513-$ 518

[14] Kantorovich V.; Pacak, K.; Pheochromocytoma and Paraganglioma, Prog. Brain Res. 2010, 182, 343-373

[15] Walther, M. M.; Keiser, H. R.; Linehan, W. M., Pheochromocytoma: Evaluation, Diagnosis, and Treatment, World J. Urol. 1999, 17, 35-39.

[16] Cole, S. W.; Korin, Y. D.; Fahey, J. L.; Zack, J. A., Norepinephrine Accelerates HIV Replication via Protein Kinase A-Dependent Effects on Cytokine Production, J Immunol. 1998, 161, 610-616. 
[17] Kanamori, T.; Funatsu, T.; Tsunoda, M.; Determination of Catecholamines and Related Compounds in Mouse Urine using Column-Switching HPLC, Analyst. 2016, 141, 2568-2573.

[18] Liu, L.; Li, Q.; Li, N.; Ling, J.; Liu, R.; Wang, Y.; Sun, L.; Chen X. H.; Bi, K., Simultaneous Determination of Catecholamines and their Metabolites Related to Alzheimer's Disease in Human Urine, J. Sep. Sci, 2011, 34, 1198-1204.

[19] Karim, M. M.; Alam, S. M.; Lee, S. H., Spectrofluorimetric Estimation of Norepinephrine using Ethylenediamine Condensation Method, J. Fluoresc. 2007, 17, 427-436

[20] Wei, F.; Xu, G.; Wu, Y.; Wang, X.; Yang, J.; Liu, L.; Hu, Q., Molecularly Imprinted Polymers on Dual-Color Quantum Dots for Simultaneous Detection of Norepinephrine and Epinephrine, Sens. Actuators B Chem. 2016, 229, 38-46.

[21] Samdani, K. J.; Samdani, J. S.; Kim, N. H.; Lee, J. H., FeMoO4 based, Enzyme-Free Electrochemical Biosensor for Ultrasensitive Detection of Norepinephrine, Biosens. Bioelectron. 2016, 81, 445-453

[22] Łuczak, T., Determination of Norepinephrine Alone and in the Presence of Ascorbic and Uric Acids Using a Gold Electrode Modified with Gold Nanoparticles and Self-Assembled Layers of meso-2,3Dimercaptosuccinic Acid, Electroanalysis 2014, 26, 1461-1470

[23] Kalimuthu, P.; John, S. A., Selective Determination of Norepinephrine in the Presence of Ascorbic and Uric Acids using an Ultrathin Polymer Film Modified Electrode, Electrochim. Acta 2011, 56, 2428-2432.

[24] Santos-Figueroa, L. E.; Moragues, M. E.; Climent, E.; Agostini, A.; Martínez-Mañez, R.; Sancenón, F., Chromogenic and Fluorogenic Chemosensors and Reagents for Anions. A Comprehensive Review of the years 2010-2011, Chem. Soc. Rev. 2013, 42, 3489-3613.

[25] Baron, R.; Zayats, M.; Willner, I., Dopamine-, L-DOPA-, Adrenaline-, and Noradrenaline-Induced Growth of Au Nanoparticles: Assays for the Detection of Neurotransmitters and of Tyrosinase Activity, Anal. Chem. 2005, 77, 1566-1571.

[26] Zhu, M.; Huang, X.; Li, J.; Shen, H., Peroxidase-Based Spectrophotometric Methods for the Determination of Ascorbic Acid, Norepinephrine, Epinephrine, Dopamine and Levodopa, Anal. Chim. Acta. 1997, 357, 261-267.

[27] Secor, K. E.; Glass, T. E., Selective Amine Recognition: Development of a Chemosensor for Dopamine and Norepinephrine, Org. Lett. 2004, 6, 3727-3730.

[28] Zhu, S.; Yang J.; Zhao, X. E.; Kong, R.; Wang, H.; You, J., Simple and Fast Determination of Catecholamines in Pharmaceutical Samples using $\mathrm{Ag}+-3,3^{\prime}, 5,5^{\prime}$-tetramethylbenzidine as a Colorimetric Probe, Anal. Methods 2015, 7, 6785-6790. 
[29] Jafarinejad, S.; Ghazi-Khansari, M.; Ghasemi F.; Sasanpour, P.; Hormozi-Nezhad, M. R., Colorimetric Fingerprints of Gold Nanorods for Discriminating Catecholamine Neurotransmitters in Urine Samples, Sci. Rep 2017, 7, 8266-8273.

[30] Menon, S.; Jose, A. R.; Jesny, S.; Kumar, K. G., A colorimetric and Fluorometric Sensor for the Determination of Norepinephrine, Anal. Methods, 2016, 8, 5801-5805.

[31] Saha, K.; Agasti, S. S.; Kim, C.; Li, X.; Rotello, V. M., Gold Nanoparticles in Chemical and Biological Sensing, Chem. Rev. 2012, 112, 2739-2779.

[32] Mayer, K. M.; Hafner, J. H., Localized Surface Plasmon Resonance Sensors, Chem. Rev. 2011, $111,3828-3857$.

[33] Wang, R.; Wang, R.; Ge, B.; Jia, X.; Li, Z.; Chang, J., Spectral Method Determination of Kanamycin Sulfate using both Gold Nanoparticles and Quantum Dots, Anal. Methods, 2013, 5, 5302 - 5308.

[34] Rawat, K. A.; Bhamore, J. R.; Singhal, R. K.; Kailasa, S. K., Microwave Assisted Synthesis of Tyrosine Protected Gold Nanoparticles for dual (Colorimetric and Fluorimetric) detection of Spermine and Spermidine in Biological Samples, Biosens. Bioelectron. 2017, 88, 71-77

[35] Martí, A.; Costero, A. M.; Gaviña, P.; Parra, M., Selective Colorimetric NO (g) Detection based on the use of Modified Gold Nanoparticles using Click Chemistry, Chem. Commun. 2015, 51, 30773079

[36] Rohit, J. V.; Kailasa, S. K., Simple and Selective Detection of Pendimethalin Herbicide in Water and Food Samples based on the Aggregation of Ractopamine-Dithiocarbamate Functionalized Gold Nanoparticles, Sens. Actuators B Chem. 2017, 245, 541-550

[37] Chen, L.; Lu, W.; Wang, X.; Chen, L., A Highly Selective and Sensitive Colorimetric Sensor for lodide Detection based on Anti-Aggregation of Gold Nanoparticles, Sens. Actuators B Chem. 2013, $182,482-488$.

[38] Kong, B.; Zhu, A.; Luo, Y.; Tian, Y.; Yu, Y.; Shi, G., Sensitive and Selective Colorimetric Visualization of Cerebral Dopamine based on Double Molecular Recognition, Angew. Chem. 2011, 123, $1877-1880$.

[39] Godoy-Reyes, T. M.; Llopis-Lorente, A.; Costero, A. M.; Sancenón, F.; Gaviña, P.; MartínezMáñez, R., Selective and Sensitive Colorimetric Detection of the Neurotransmitter Serotonin based on the Aggregation of Bifunctionalized Gold Nanoparticles, Sens. Actuators B Chem. 2018, 258, 829-835.

[40] Agami, C.; Rizk, T., Role of Solvent on the Diastereoselectivity of Oxazolidine Formation from (-)Ephedrine, J. Chem. Soc., Chem. Commun. 1983, 0, 1485-1486.

[41] Juhász, M.; Lázár, L.; Fülöp, F., Substituent Effects in the Ring-Chain Tautomerism of 4-Alkyl-2-aryl substituted Oxazolidines and Tetrahydro-1,3-oxazines, J. Heterocycl Chem. 2007, 44, 1465-1473. 
[42] Freeman, R.; Elbaz, J.; Gill, R.; Zayats, M.; Willner, I., Analysis of Dopamine and Tyrosinase Activity on Ion-Sensitive Field-Effect Transistor (ISFET) Devices, Chem. Eur. J. 2007, 13, 72887293

[43] Wu, W.; Zhu, H.; Fan, L.; Liu, D.; Renneberg R.; Yang, S., Sensitive Dopamine Recognition by Boronic Acid Functionalized Multi-Walled Carbon Nanotubes, Chem. Commun. 2007, 23, 23452347.

[44] Zhao, P.; Li N.; Astruc, D., State of the Art in Gold Nanoparticle Synthesis, Coord. Chem. Rev. 2013, 257, 638-665.

[45] Turkevich, J.; Stevenson P. C.; Hillier, J., A Study of the Nucleation and Growth Processes in the Synthesis of Colloidal Gold, Discuss. Faraday Soc. 1951, 11, 55-75.

[46] Frens, G., Controlled Nucleation for the Regulation of the Particle Size in Monodisperse Gold Suspensions, Nature-Phys Sci 1973, 241, 20-22.

[47] Haiss, W.; Thanh, N. T. K.; Aveyard J.; Fernig, D. G., Determination of Size and Concentration of Gold Nanoparticles from UV- Vis Spectra, Anal. Chem. 2007, 79, 4215-4221

[48] Lin, S.-Y.; Tsai, Y.-T.; Chen, C.-C.; Lin C.-M.; Chen, C.-H., Two-step Functionalization of Neutral and Positively Charged Thiols onto Citrate-Stabilized Au Nanoparticles, J. Phys. Chem. B. 2004, $108,2134-2139$.

[49] Liu, X.; Atwater, M.; Wang J.; Huo, Q., Extinction Coefficient of Gold Nanoparticles with Different Sizes and Different Capping Ligands, Colloids Surf. B. 2007, 58, 3-7.

[50] Zhang, Y.; Li, B.; Chen, X., Simple and Sensitive Detection of Dopamine in the presence of High Concentration of Ascorbic Acid using Gold Nanoparticles as Colorimetric Probes, Microchim Acta, 2010, 168, 107-113.

[51] Fischbach, F. T.; Dunning, M. B., A Manual of Laboratory and Diagnostic Tests. 8th Edition. Walters Kluwer Health/ Lippincott Williams \& Wilkins, 2009

[52] Cerilliant Analytical Reference Standards, https://www.cerilliant.com/shoponline/Item_Details.aspx?itemno=1cedba31-c7f4-4ab1-ba9fa02f3328d204\&item=S-020, (accessed May 2018). 
Table of Contents graphic

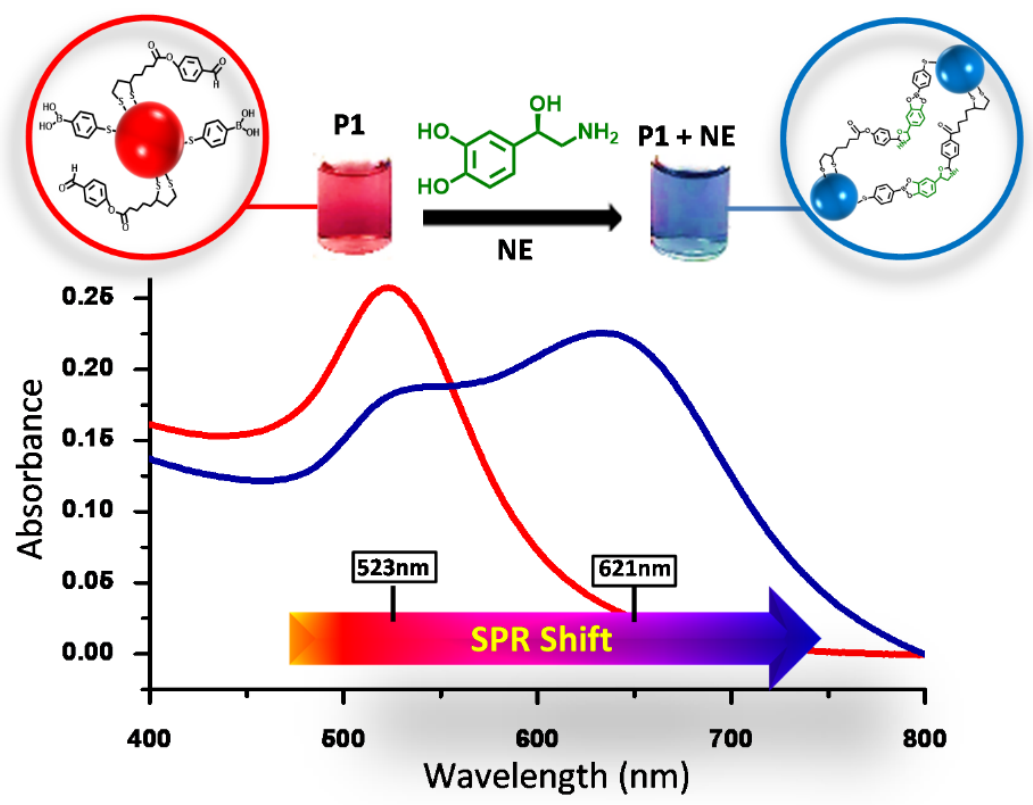

